\title{
La participación estudiantil como proceso de inclusión educativa
}

\section{Student participation as a process of educational inclusion}

\author{
RENÉ VALDÉS* \\ DOMINIQUE MANGHI ${ }^{*}$ \\ GERARDO GODOY ${ }^{* * *}$
}

El objetivo de este estudio es analizar los procesos de participación estudiantil en función de la valoración de los estudiantes en el espacio escolar. Mediante un enfoque etnográfico, se aplicó una técnica participativa a estudiantes de seis escuelas chilenas. Los resultados señalan que estos demandan una mayor participación en los espacios escolares. Desean participar dentro y fuera de la escuela y reinventar las prácticas educativas más tradicionales. Su propósito sería desarrollarse personalmente, mejorar la experiencia escolar y tomar decisiones. Además, valoran en forma positiva la capacidad e integridad de sus pares y de manera negativa la de los adultos.

Through an ethnographic approach, a participatory technique was carried out on students from six Chilean schools. The results indicate that students demand greater participation in school spaces. They want to participate in and out of school and reinvent the most traditional educational practices. The purpose of their participation would be to develop personally, improve the school experience and make decisions. In addition, they positively value the capacity and integrity of their peers and negatively that of adults.
Palabras clave:

participación estudiantil, inclusión educativa, investigación participativa, etnografía, educación pública chilena

\section{Keywords:} student participation, educational inclusion, participatory research, ethnography, Chilean public education

Recibido: 17 de febrero de 2020 | Aceptado para su publicación: 8 de septiembre de 2020| Fecha de publicación: 25 de septiembre de 2020

Recuperado de: https://sinectica.iteso.mx/index.php/SINECTICA/article/view/1096 doi: 10.31391/S2007-7033(2020)0055-008

\footnotetext{
* Doctor en Psicología. Docente de la Escuela de Pedagogía de la Pontificia Universidad Católica de Valparaíso, Chile, e investigador posdoctorando en la Facultad de Educación y Ciencias Sociales de la Universidad Andrés Bello. Líneas de investigación: liderazgo escolar, inclusión e interculturalidad. Correo electrónico:revalmorales@gmail.com/https://orcid. org/0000-0003-4242-9748

** Doctora en Lingüística. Académica de la Escuela de Pedagogía de la Pontificia Universidad Católica de Valparaíso e investigadora del Centro de Investigación para la Educación Inclusiva. Líneas de investigación: prácticas educativas a través de la teoría de la semiótica social y metodologías etnográficas y participativas. Correo electrónico: dominique.manghi@pucv. cl/https://orcid.org/0000-0002-0278-9899

*** Magíster en Lingüística y estudiante de doctorado en la Pontificia Universidad Católica de Chile. Investigador en el Centro de Investigación para la Educación Inclusiva. Líneas de investigación: inclusión escolar, discursos críticos sobre la discapacidad, y discapacidad a través de la teoría de la semiótica social. Correo electrónico: gerardo.godoy@pucv.cl/ https://orcid.org/0000-0002-2960-3548
}

| Esta investigación ha sido financiada por ANID-PIA CIE 160009 


\section{INTRODUCCIÓN}

$\mathrm{E}$ s posible encontrar un consenso en el ámbito educativo de que las escuelas deben ir avanzando hacia un modelo de escuela inclusiva, entendiendo la inclusión como un proceso que asegura oportunidades equivalentes para todo el alumnado (López, 2012), valora la diversidad e implica la presencia, el aprendizaje y la participación de todas y todos los estudiantes en la escuela, no solo de aquellos etiquetados históricamente como con necesidades educativas especiales (Unesco, 2018). En el contexto actual, dada la impronta neoliberal de la educación chilena y los efectos de la rendición de cuentas en las prácticas educativas (Sisto, 2019), es necesario precisar que, además, este proceso debiera ser colectivo, interdisciplinario y que interpela a toda la sociedad en su conjunto.

A nivel internacional han sido variadas las iniciativas políticas para avanzar en inclusión. Desde la declaración de Salamanca (Unesco, 1994) hasta las diversas rutas de acción de organismos internacionales (Unesco, OCDE, UNICEF, Cepal, entre otros), el propósito ha sido propender a la creación de sistemas escolares inclusivos que disminuyan la segregación y la exclusión escolar. Sin embargo, esto implica necesariamente una acción conjunta, un cuestionamiento a las políticas neoliberales y la idea de que la inclusión no se juega solo en la escuela, sino que es a través de ella y en sincronía con otras medidas sociales.

En el plano nacional, en estas últimas tres décadas, el modelo económico y social ha influido en mutaciones culturales radicales en torno a la escuela y a las familias. Reyes, Cornejo, Arévalo y Sánchez (2010) las sintetizan en la masificación de la escolaridad; las transformaciones en el mercado del trabajo y su efecto en patrones de formas de vincularse en las familias y las comunidades; la explosión del acceso a nuevas "fuentes de socialización", como internet y redes sociales; y la crisis de las certezas de la escuela. Ejemplo de esto último es el efecto de las pruebas estandarizadas que evalúan aprendizajes curriculares, con la consecuente sobrecarga para los actores escolares y los premios o sanciones para los centros educativos.

En este contexto, las políticas educativas chilenas, como la ley 20201 de los Programas de Integración Escolar (2007), la ley 20422 de Inclusión Social (2010) y la ley 20845 de Inclusión Escolar (2015), a pesar de las diferencias en sus enfoques (Ramírez y Valdés, 2019), marcan una agenda social que se desarrolla en un proceso constante de despolitización y quiebre del tejido social, y estimula el bienestar individual a través del consumo (Cornejo y Reyes, 2008). Esto es parte del contexto inmediato donde se dan, o no, prácticas educativas que promueven la participación del estudiantado.

La evidencia plantea que la participación del alumnado dentro de las escuelas, entendida como un proceso de inclusión y que implica considerar sus voces para repensar las prácticas educativas, ha estado ausente en estas últimas y, por lo tanto, se ha vuelto un proceso complejo (Chávez y Vergara, 2017; Poblete y Galaz, 2017; Thousand, Villa y Nevin, 2015; Vergara, Peña, Chávez y Vergara, 2015; Nieto y Portela, 2008). Lo anterior resulta un punto crítico, pues, desde un enfoque inclusivo, los estudiantes son sujetos de derecho y deben ser actores socialmente validados dentro del espacio social, por lo que resulta esencial no solo escuchar sus posicionamientos frente a su experiencia escolar, sino tomar medidas a partir de ellos. 
Por esta razón, el objetivo de este estudio es analizar los procesos de participación estudiantil en función de la valoración que realizan los estudiantes de su experiencia escolar.

\section{DESARROLLO}

Políticas de inclusión educativa: el caso chileno

Desde la década de los setenta, Chile ha instalado las estructuras de un modelo educativo basado en principios de mercado (Bellei, 2015; Sisto, 2019), y se ha comprometido a la vez con la inclusión y los derechos educativos. Desde la creación en esos años de la modalidad de educación especial, pasando por la ley 9284 de Integración Social de las Personas con Discapacidad (1994), el decreto 291 que regula los grupos diferenciales (1999), una política nacional de educación especial (Ministerio de Educación, 2005), la ley 20201 de los Programas de Integración Escolar (2007), la ley 20248 de Subvención Escolar Preferencial (2008), la ley 20422 de Inclusión Social (2010) y la ley 20845 de Inclusión Escolar (2015), se ha construido de manera progresiva un modo particular de entender la inclusión en el sistema chileno.

En este contexto de políticas públicas, López et al. (2018) identifican y analizan tres nudos críticos que dificultan la instalación de la inclusión educativa en Chile. El primero es el modelo educativo basado en principios de mercado, como la competitividad y la libertad de enseñanza; el segundo, la tensión entre la lógica de la integración e inclusión educativa en la cual las diferencias son fuente de discriminación. El tercero, la arquitectura educacional basada en la perspectiva de la rendición de cuentas individual con foco en las pruebas de alto rendimiento, que busca que las escuelas públicas compitan por recursos financieros, condicionados a la demostración de evidencias de productividad y eficiencia. Esta arquitectura es criticada dado que restringe la noción de lo que el país entiende por calidad de la educación y deja fuera del ejercicio pedagógico otras áreas no evaluadas como la participación escolar.

En los últimos años, la evaluación de las escuelas a través del Sistema Nacional de Aseguramiento de la Calidad de la Educación (ley 20529, 2011) ha incorporado otros indicadores de calidad o "indicadores no académicos" como clima escolar o ciudadanía, los cuales componen el 33\% de la evaluación, mientras que el rendimiento académico implica el 67\%. Este último condiciona el cierre de escuelas públicas. A esto se suman otras características que vuelven más sensible el problema educativo, como la disminuida innovación en educación, bajo liderazgo escolar y la instauración de mandatos en el sistema escolar sin la participación activa de los profesores (Bellei, 2015).

Según Lerena y Trejos (2015), las consecuencias de crear un sistema escolar basado en principios de mercado y con foco en pruebas estandarizas han sido claras y sus resultados han hecho más dificultoso el camino hacia el desarrollo de procesos inclusivos, como es el caso de la participación estudiantil.

\section{La participación estudiantil como proceso de inclusión}

Consideramos que atender la voz estudiantil es una práctica de inclusión y, a la vez, una práctica de resistencia hacia enfoques educativos neoliberales y adultocéntricos. Por 
una parte, esto implica un posicionamiento de los actores educativos frente a las demandas de la educación de mercado. No es solo considerar la voz estudiantil para cumplir el checklist administrativo que refuerza el statu quo (Slee, 2013); más bien debería ser un compromiso genuino con los derechos educativos, la justicia y la democracia. Según Fielding (2011), hay que desarrollar una educación centrada en la persona, lo que no se demuestra en el éxito académico escolar, sino en entender la experiencia escolar como cocreadora de las concepciones de aprendizaje.

Lo anterior coincide con la idea de dejar de concebir la infancia únicamente como un periodo en el que se llega a ser alguien, y considerarla una etapa en la que se está siendo alguien (becoming/being); es decir, no como una fase transicional de preparación para la adultez, en la cual el niño refleja un futuro adulto, pero sin el mismo estatus; más bien se aboga por el reconocimiento del aporte presente del niño como un miembro integral de la sociedad (Huang, 2019). Estos planteamientos son coherentes con los de los nuevos estudios de la infancia: ser niño es ser parte permanente de una estructura social. Desde la perspectiva sociológica, niños, niñas y jóvenes se ven constantemente afectados por las estructuras políticas y económicas, y deben ser configurados como actores sociales y no como instrumentos del orden social (Gaitán, 2006).

Según Lin (2013), la participación escolar es el fundamento principal de una democracia y una experiencia integral. Las escuelas son espacios públicos donde debe ocurrir la acción colectiva y, por lo tanto, el desarrollo de la voz estudiantil. La escuela debe ser un lugar donde se configure la infancia como estructura de participación política y ciudadanía activa, razón por la cual debe generar acciones que posibiliten el desarrollo integral de sus estudiantes.

Según Graham et al. (2018), la participación de los estudiantes en las escuelas mejora servicios, seguridad, protección, participación y relaciones entre adultos y jóvenes. Además, incluye el involucramiento estudiantil activo en la toma de decisiones en la escuela, aprendizaje, educación y vida como miembros de la escuela y comunidad. Desde estas concepciones, la participación no sería necesariamente un proceso académico, aunque también lo es, sino que participar en la escuela sería una tarea que refleja el cumplimiento de derechos educativos y de justicia social.

\section{Los dilemas de la participación estudiantil}

En este trabajo nos acogemos a la idea de que la participación estudiantil es un proceso de y para la inclusión educativa. Sostenemos que la inclusión ha sido pensada desde el mundo adulto y, por lo tanto, necesitamos escuchar las voces de los estudiantes para pensarla en conjunto, en un sentido amplio y, así, mejorar la experiencia escolar integral de la comunidad educativa. En ese escenario, podemos problematizar la participación estudiantil en la escuela considerando tres dilemas:

\section{La participación como proceso neoliberal e instrumental}

La participación puede tener distintos objetivos, algunos de ellos muy instrumentales; por ejemplo, subir el puntaje en pruebas estandarizadas o mantener el control 
de las disposiciones del proyecto educativo. A pesar de que, en nombre de la inclusión y la convivencia, la política educativa ha promovido la participación estudiantil en la escuela, esta se ha vuelto un proceso instrumental. Incluso en las escuelas con alta calidad de convivencia escolar, los adultos deciden quiénes participan, cómo se participa y para qué se participa. La participación escolar sería un mandato de los adultos y que pronto configuraría en los estudiantes una subjetividad pasiva y dependiente (Ascorra, López y Urbina, 2016).

Según Fielding (2011), la participación implica un posicionamiento político, ya que es posible encontrar una voz neoliberal de participación. Se puede promover la participación en la escuela para aumentar la obediencia, la productividad o la competencia, incluso la segregación, como es el caso de los liceos de excelencia chilenos. Entonces, se puede abogar por la participación en un contexto educativo sin que, necesariamente, las acciones participativas se orienten por principios inclusivos. Una participación genuina implica consensuar un punto de vista hacia modelos dominantes. Según LayLisboa, Araya-Bolvarán, Marabolí-Garay, Olivero-Tapia y Santander-Andrade (2018), los docentes chilenos posibilitan espacios de participación, pero lo hacen desde un paternalismo moderno. No sería solo participar para estar mejor en la escuela, aunque también lo es; se trataría de participar para transformar la escuela.

\section{La participación como proceso impuesto, obligatorio y bajo demanda}

Los documentos de política internacional han influido en las políticas nacionales para comprometer a Chile en el avance de una educación inclusiva. Documentos oficiales recientes, como las orientaciones para la construcción de comunidades educativas inclusivas o la política de convivencia escolar a través de los consejos escolares, plantean la participación como un requisito exigido a las escuelas para sus procesos de inclusión. Estas políticas actúan sobre las escuelas de administración municipal en peligro de extinción por matrícula insuficiente, o por no superar la categorización que las pone en permanente amenaza de cierre, mientras luchan por reencontrar un sentido, sin puntos de diálogo, los cuales han sido desplazados por espacios de gestión (Assaél, Acuña, Contreras y Corvalán, 2014).

En este contexto, ser una escuela inclusiva es una exigencia. La teoría define la participación escolar como un valor inclusivo (Booth y Ainscow, 2015): "Estar y colaborar con otros. Hace mención a la tarea de participar en las decisiones sobre la propia vida, incluida la educación y enlaza con las ideas de democracia y libertad" (p. 27). Desde el índice de inclusión (Booth y Ainscow, 2015), la participación sería propositiva, porque supone un conjunto de acciones que implican colaboración y democracia.

El estudio chileno de Albornoz, Silva y López (2015) define la participación como implicarse activamente en la toma de decisiones; reconocer y valorar una variedad de identidades, que todos sean aceptados por quienes son. La noción de involucramiento activo incluye acceso (estar ahí), colaboración (aprender juntos) y diversidad (reconocimiento y aceptación). Tanto estos autores como Booth y Ainscow (2015) configuran la participación como un proceso que permite avanzar en inclusión educativa. La pregunta que orienta este dilema sería cómo un proceso propositivo, democrático y libre puede ser configurado como un proceso impuesto (López, 2012), como ocurre en el caso chileno. 


\section{La participación como proceso selectivo}

Uno de los problemas más presentes en la escuela es que solo algunos participan; lo observamos en actos escolares o dentro de las clases. Los adultos eligen a los que son más dignos de participación: los ordenados, los que tienen buenas notas o los disciplinados. La participación parece un premio que se gana y no un derecho a tener voz.

Estudios chilenos, como el de Ramírez (2020), indican que los estudiantes legitiman la participación dentro de la escuela como algo relevante para su bienestar. No obstante, existen estudiantes, como los que tienen buen rendimiento, que son más escuchados y considerados que aquellos con peores calificaciones. Este aspecto es compartido por Valdés (2019), quien señala que, sin importar el nivel de culturas inclusivas que tengan las escuelas, la decisión de participar la toman los adultos, al seleccionar a quienes poseen mayor dominio discursivo, punto coincidente con Ascorra, López y Urbina (2016), que hablan de una participación selectiva y tutelada por adultos, incluso en escuelas con buena convivencia escolar.

Según Fielding (2011), han aumentado los estudios sobre participación y voz estudiantil, pero solo pensando en algunos grupos. Existen alumnos sin voz, por ejemplo, los que pertenecen a los programas de educación especial. Para este autor, la paradoja es que, al centrarse en algunos, se sofoca la diferencia y se atrapan a jóvenes y adultos en una cultura de la mayoría. Considerar la voz únicamente de algunos sería muy riesgoso, ya que aparenta preocupación e interés, pero convierte cualquier aspiración genuina de inclusión en una parodia corrosiva.

Algo común a estos tres dilemas es que la mayoría de las decisiones dentro de la escuela son tomadas por adultos y desde diversas posiciones de poder. Esto implica, según Liebel (2007), un paternalismo, en cuanto se asume desde antes lo que es mejor para el alumnado. La participación escolar sería, entonces, un proceso adultocéntrico y paternalista, lo que nos lleva al terreno de la educación. Los defensores del enfoque tradicional concuerdan en que el objetivo de la educación es desarrollar al adulto en potencia, a partir de hechos y conocimientos provenientes del mundo adulto, mientras que otros proponen colocar al niño al centro y responder a las necesidades de su desarrollo (Huang, 2019).

Sea de una u otra manera, el sentido de los saberes, las situaciones y los aprendizajes escolares se construye con base en una cultura. Por lo tanto, si la participación requiere la figura central del estudiante, habría que repensar el oficio de alumno, el rol de la escuela y, radicalmente, el oficio del maestro (Perrenoud, 2006). Compartimos de manera plena esta noción. Sin embargo, es necesario profundizar en esta idea y señalar que la participación, como proceso, debe ser inclusiva y considerar la voz del estudiantado para su permanencia y legitimidad.

Por lo tanto, entendemos la participación escolar como un proceso inclusivo en sí mismo, pero que posibilita la inclusión en tanto permite la presencia en los diversos espacios escolares y la colaboración en diferentes niveles y entre distintos actores educativos, la cual es una acción voluntaria que incluye autonomía y libertad. Del mismo modo, y de manera situada, reconocemos los problemas que existen para escuchar las voces de los estudiantes, promover la participación y hacerla permanente en el tiempo. 


\section{DISCURSO Y VALORACIÓN}

Una de las formas de abordar las voces de quienes participan en las comunidades educativas es mediante los discursos que producen. Los discursos en este contexto se entienden como lenguaje en uso, es decir, la producción textual que realiza una persona en un contexto determinado (Fairclough, 2013). Los discursos tienen el potencial de dar cuenta de la experiencia en torno a los sucesos y actores representados, además de un posicionamiento intersubjetivo (Martin \& Rose, 2007) que refleja la voz de quien se expresa.

El posicionamiento intersubjetivo corresponde a la negociación de las valoraciones en un discurso determinado (Martin \& Rose, 2007); por ejemplo, un sujeto podría valorar un hecho como positivo o negativo y posicionarse en uno u otro sentido, para lo cual deberá utilizar, necesariamente, ciertos recursos lingüísticos que permiten expresar y dar cuenta de emociones, juicios o apreciaciones, así como certeza o duda, junto con la negación o inclusión de actores en un relato. Así, el análisis del discurso contribuye a mirar cómo los productores de un discurso usan los recursos lingüísticos para tomar una posición sobre personas, cosas o acontecimientos.

Unas de las herramienta teóricas y metodológicas para dar cuenta de la intersubjetividad en el discurso corresponde al sistema de valoración (Martin \& White, 2005), el cual se enmarca en la teoría lingüística sistémica funcional (Halliday \& Matthiessen, 2014), que plantea que los significados se expresan de modo simultáneo de tres formas, denominadas metafunciones. La primera es la metafunción ideacional, que permite la representación de la experiencia; la segunda es la metafunción interpersonal, que facilita la expresión de relaciones interpersonales e intersubjetivas; la tercera es la metafunción textual, la cual organiza al texto para que tenga un significado con sentido. El sistema de valoración da cuenta de los significados interpersonales en el discurso por medio de las actitudes de los hablantes/ escritores (Martin \& White, 2005).

El modelo está conformado por tres ámbitos semánticos: actitud, compromiso, gradación. La actitud tiene como elemento central los sentimientos que se negocian en un discurso y se expresan de tres maneras: como afectos por parte de actores humanos que se presentan como productores o sintientes de una emoción; como juicios sobre el comportamiento de las personas, su capacidad para llevar a cabo una actividad, su integridad ética o de valor, así como la normalidad y determinación que pueda tener un actor en un discurso particular; y como apreciaciones respecto a ideas, cosas o hecho. El conjunto de estas formas de expresión de los sentimientos configura el ámbito semántico de la actitud y sus tres subsistemas: afecto, juicio y apreciación (Martin \& White, 2005; Oteíza, 2019).

La actitud, a su vez, puede ser expresada en una escala de gradación. Por ejemplo, un afecto puede ser presentado con niveles de intensidad: contento, feliz, dichoso. Los niveles de intensidad de una valoración aportan a la construcción del significado interpersonal a través de, por ejemplo, la modalización de una expresión: "El estudiante es un poco enojón". Esta posibilidad de escalar de una actitud configura el subsistema de gradación (Martin \& White, 2005; Oteíza, 2019).

Por último, los discursos presentan diferentes actores que pueden traer voces de otros a sus relatos. Como plantea Bajtín (2015), un discurso nunca es monológico; 
siempre está constituido por una polifonía de voces; sin embargo, un hablante/escritor puede elegir ocultar o visibilizar estas voces. Esta elección en el modo de mostrar la visibilización u ocultamiento de voces implica un tipo de compromiso sobre lo que se relata; por ejemplo, participar es positivo/participar podría ser positivo. Este posicionamiento se puede dar desde el subsistema de compromiso con los criterios de monoglosia (cierre de voces) y heteroglosia (apertura de voces).

Por lo tanto, el abordaje de la intersubjetividad en los discursos constituye un posicionamiento teórico y una propuesta metodológica. Es un posicionamiento al abordar el discurso desde una dimensión lingüística; no como práctica social en sí misma, sino como parte de ella, y es una propuesta metodológica al requerir el encuentro entre un análisis de contenido con los criterios del sistema de valoración, los cuales requieren un puente dialógico para construir las interpretaciones de los análisis a través de estas dos miradas complementarias.

Con base en este punto de vista, es relevante escuchar a los estudiantes desde sus propias valoraciones, motivo por el cual el objetivo de este trabajo es analizar los procesos de participación escolar en función de la valoración de los estudiantes en el espacio escolar.

\section{Metodología}

\section{Diseño}

Este estudio deriva de una investigación mayor cuyo propósito es analizar la participación de niños y jóvenes de escuelas públicas chilenas en las prácticas educativas y procesos inclusivos a partir de sus experiencias y valoraciones. Con un diseño cualitativo y mediante un enfoque etnográfico, realizamos entrevistas individuales, grupos focales, metodologías participativas, observaciones participantes y revisión de documentos clave en nueve escuelas de tres regiones de Chile. Este trabajo reporta los resultados de las metodologías participativas aplicadas en estudiantes de seis centros escolares entre agosto de 2017 y julio de 2018.

\section{Participantes}

En total, sesenta estudiantes de enseñanza primaria y secundaria participaron, diez por cada una de las seis escuelas chilenas. Dos centros pertenecientes a la comuna de Alto Hospicio del norte de Chile; dos, a la Región Metropolitana, capital y zona central; y dos de la comuna de Viña del Mar, ciudad que se encuentra en el litoral central. El criterio para seleccionar los establecimientos escolares consideró su índice de vulnerabilidad y que tuvieran la inclusión como sello y propósito institucional. Por lo tanto, el muestreo fue teórico e intencionado (Strauss \& Corbin, 1994), ya que el valor de los datos cualitativos no radica en su representación, sino en la profundidad local del conocimiento, que, para este caso, se encuentra en tres zonas geográficas que son distintas entre sí en cuanto a sus aspectos demográficos y económicos. 


\section{Trabajo de campo}

Para la producción de información, utilizamos la técnica de grupo focal (Canales, 2006). Los criterios para la conformación heterogénea de los grupos consideraron máximo diez estudiantes por escuela con distribución equitativa de género, presencia de estudiantes que pertenecieran al Programa de Integración Escolar, de estudiantes migrantes, estudiantes del centro general de alumnos, un estudiante nuevo y uno que llevara más de cuatro años en la escuela. Cada grupo abarcaba los niveles primarios (cuarto a sexto básico) y secundarios (octavo a segundo medio). Organizamos seis grupos focales, uno por escuela.

Utilizamos una pauta de trabajo inspirada en metodologías participativas de investigación, que consistió en dos etapas: una, presentación y uso de superpoderes: cada estudiante se presenta frente a sus compañeros y elige un objeto que representará un superpoder; luego señala su superpoder imaginado y para qué lo utilizaría; y dos, creación del horario escolar: frente a un horario semanal en formato gigante, en grupos pequeños, acuerdan cómo les gustaría su semana de clases de lunes a viernes.

La instrucción consistía en soñar su escuela con sus propios horarios, formas de participar, sus actividades y aprendizajes para que todos y todas pudieran sentirse cómodos y felices. Los integrantes del grupo no se conocían entre sí y era necesario que negociaran qué harían en la semana, cómo lo harían y quiénes participarían. La actividad duró sesenta minutos aproximadamente; el monitor acompañaba con preguntas modeladoras de la experiencia escolar, y se registró de manera audiovisual. El análisis de los datos se llevó a cabo en esta segunda parte: creación del horarios soñado.

\section{Técnicas de análisis}

Para examinar los datos producidos, recurrimos al análisis cualitativo secuencial con una primera fase de contenido y una segunda fase discursiva; es decir, primero analizamos las ideas presentes en los relatos de los estudiantes y, luego, sobre una primera versión de categorías, consideramos el posicionamiento valorativo de los estudiantes.

En la primera fase realizamos un análisis de contenido temático (Flick, 2012) que permitió gestionar el procedimiento inductivo para el tratamiento de los datos, el cual consistió en:

- La preparación de documentos primarios. El material producido en los grupos focales fue transcrito y luego asignado a una unidad hermenéutica.

- Codificación. Mediante la lectura reiterada del material, este es segmentado en unidades de significado que refieren y describen acciones y discursos de los estudiantes a partir de los cuales elaboramos un libro de códigos. De la codificación de las diversas prácticas, emergieron las categorías consensuadas de resultados y aseguramos la validez con la revisión del objetivo respecto a los códigos obtenidos (Barrios, Gutiérrez, Mayorga y Urrutia, 2014).

- Categorización. La lectura sistémica de los códigos permitió agruparlos 
y comprender los patrones de significado comunes al tipo de escuelas que configuraban los estudiantes en sus grupos focales. Organizamos la diversidad de prácticas, discursos y sueños con tres preguntas orientadoras: ¿con quiénes desean participar los estudiantes?, ¿cómo desean participar? y ¿para qué desean participar?

En una segunda fase llevamos a cabo un análisis discursivo valorativo. Las citas de las categorías que responden a las preguntas orientadoras constituyen el corpus textual que se analiza, discursivamente, para revelar las valoraciones de los estudiantes. Como mencionamos en el marco conceptual, el posicionamiento valorativo o intersubjetivo se enmarca en una noción discursiva desde la lingüística que aporta al análisis social del fenómeno estudiado. Los recursos que ofrece el sistema de valoración (Martin \& White, 2005) son sus tres subsistemas de análisis: actitud, gradación y compromiso. En la tabla ejemplificamos cada uno de los subsistemas de actitud.

Tabla. Subsistemas de actitud pertenecientes al sistema de valoración

\begin{tabular}{|c|c|c|}
\hline \multicolumn{3}{|c|}{ Actitud } \\
\hline Subsistema & Categoría & Ejemplo \\
\hline \multirow{4}{*}{$\begin{array}{l}\text { Afecto } \\
\text { (da cuenta de los sentimientos como po- } \\
\text { sitivos o negativos, e indica una dispo- } \\
\text { sición emocional hacia personas, cosas, } \\
\text { situaciones o sucesos) }\end{array}$} & inclinación & "yo quiero helado" \\
\hline & satisfacción & "me gusta el helado" \\
\hline & felicidad & $\begin{array}{l}\text { "el invierno me } \\
\text { alegra" }\end{array}$ \\
\hline & seguridad & "él confía en eso" \\
\hline \multirow{5}{*}{$\begin{array}{c}\text { Juicio } \\
\text { (da cuenta del posicionamiento sobre la } \\
\text { institucionalización de las emociones, } \\
\text { como las normas o leyes) }\end{array}$} & capacidad & "ella puede hacerlo" \\
\hline & normalidad & "él juega raro" \\
\hline & tenacidad & "ellos se rinden" \\
\hline & veracidad & "ella es honesta" \\
\hline & integridad & "él es generoso" \\
\hline \multirow{4}{*}{$\begin{array}{l}\text { Apreciación } \\
\text { (da cuenta de la valoración de objetos, } \\
\text { ideas o fenómenos naturales) }\end{array}$} & reacción & "el juego es divertido" \\
\hline & composición & "el juego es simple" \\
\hline & valuación & $\begin{array}{l}\text { "el juego es } \\
\text { importante" }\end{array}$ \\
\hline & integridad & "jugar es bueno" \\
\hline
\end{tabular}

Fuente: Elaboración con base en Martin y White (2005).

La actitud puede presentarse de manera inscrita o evocada, es decir, explícita o implícita. Además, la actitud se expresa y analiza a través de sus patrones: la relación 
que se configura entre las valoraciones a lo largo del despliegue del texto. El segundo subsistema es el de gradación, que permite construir una relación escalar de las actitudes de fuerza o foco. La fuerza intensifica o disminuye el significado "más bello/es algo bello", mientras que el foco indica la prototipicidad de un concepto o cosa: "es un verdadero estudiante/es casi un estudiante".

El último subsistema es el de compromiso, que da cuenta de la apertura o cierre de posibilidades de encontrar diversidad de voces en un discurso determinado. El compromiso se expresa por medio de distintos recursos lingüísticos; en la presentación de ejemplos del corpus indicaremos la integración o exclusión de voces cuando sea pertinente.

El criterio de calidad científica para la investigación cualitativa lo aporta la autenticidad (Flick, 2012) y la participación, que muestran distintas realidades sobre la problemática de estudio que ofrecen una comprensión más sofisticada de la realidad. Este criterio se observa en los hallazgos y fue confirmado mediante una triangulación metodológica al interior del grupo de investigación responsable de la producción y análisis de datos (Denzin \& Lincoln, 2003).

\section{Consideraciones éticas}

Para el desarrollo de las técnicas del trabajo etnográfico, solicitamos autorizaciones a los directores de escuelas, consentimientos informados a adultos y asentimientos informados a estudiantes, todos ellos visados por el comité de bioética de la universidad patrocinadora.

\section{RESULTADOS}

Estos se organizan en dos niveles: uno de tipo descriptivo, que sistematiza los hallazgos según las preguntas orientadoras del análisis de contenido; es decir, presenta categorías que surgen de la decodificación no emergente. Las citas de estas categorías se analizan discursivamente, lo que revela los posicionamientos valorativos e intersubjetivos de los estudiantes acerca de sus procesos de participación escolar. El segundo nivel es explicativo; presenta el levantamiento de categorías finales producto del análisis de contenido y del zoom valorativo. En ambos niveles recurrimos a ejemplos concretos del corpus textual examinado.

Primer nivel: dimensión de contenido y valoración de las voces de los estudiantes. ¿Con quiénes desean participar los estudiantes?

Los estudiantes de primaria y secundaria del estudio, desde sus diversas experiencias, trayectorias y centros escolares, sueñan con una escuela amplia. Una amplitud no solo en cuanto a los espacios físicos, sino en las relaciones interpersonales. Según el análisis de contenido, al pensar en una escuela ideal, los estudiantes desean participar con sus compañeros, profesores y actores externos que no pertenecen necesariamente al ámbito educativo. 
Respecto a los compañeros, los actores mencionados para liderar y participar en la escuela son los mismos estudiantes; es decir, los participantes se visualizan a sí mismos como actores capaces y protagónicos en la toma de decisiones. A su vez, los estudiantes señalan que pueden realizar actividades de manera autónoma (ejemplo 1) y que se ven a sí mismos como potenciales enseñantes (ejemplo 2). Las siguientes citas son ilustrativas del rol protagónico y gestor que conciben los estudiantes sobre ellos:

Ejemplo 1:

Las actividades las podemos dividir entre alumnos [a] grandes y [b] chicos. También hay actividades que pueden hacerse solas (grupo focal, escuela 2).

Ejemplo 2:

Podríamos [c] enseñarle a la profesora a [d] relajarse (grupo focal, escuela 5).

Ejemplo 3:

Monitor: ¿Quién estaría a cargo de organizar esta actividad [sala de juegos]?

Estudiante: Nosotros [estamos a cargo], los presidentes del curso, y los más chicos de tercero para abajo, como [e] no tienen presidente, que sean los profesores o que [f] elijan a algún [compañero] (grupo focal, escuela 4).

Además de la lectura desde el análisis de contenido, en los ejemplos 1 y 3 apreciamos algunas instancias valorativas acerca de los roles de los estudiantes, quienes diferencian entre compañeros [a] de educación secundaria ("grandes") y [b] los de primaria ("pequeños"). Un posicionamiento clave en torno a estos actores corresponde a los juicios negativos de capacidad sobre estudiantes de educación primaria o básica, que son valorados de forma negativa al no tener representatividad formal, pero identificados en la misma jerarquía que los de secundaria al expresar su posibilidad de participación y elegir un compañero/a como representante en los procesos participativos [f]. En el ejemplo 2, los estudiantes se autovaloran cargados de capacidad en función de su posibilidad de enseñar y apoyar a los docentes; del mismo modo, la capacidad de hacer cosas aparece junto con autodefinirse con integridad de valor $[\mathrm{d}]$ :

Ejemplo 4:

Monitor: ¿Y quién va a cocinar?

Estudiantes [todos]: ¡nosotros!

Estudiante 1: [g] Yo mismo lo hago, nosotros mismos cocinamos.

Monitor: ¿Y le cocinarían a todo el colegio o a su curso?

Estudiante 1, 2 y 3: A todo el curso.

Estudiante 3: Yo como soy [h] generoso a todo el colegio (grupo focal, escuela 4). 
El ejemplo 4 refleja una valoración que aparece a lo largo del corpus analizado, los estudiantes se presentan como capaces de realizar una acción, en este caso el cocinar. Esta acción, a su vez, se aumenta en gradación a través de la reiteración del participante que ejecuta la acción y su posterior intensificación "[g] yo mismo < nosotros mismos< a todo el curso < a todo el colegio". En el mismo enunciado, un estudiante cierra la interacción expresando un valor consensuado por sus compañeros [h]: generosidad; esta expresión corresponde, desde esta perspectiva de análisis, a un juicio positivo de integridad, ya que enuncia un valor deseable en el espacio social donde se desenvuelven los participantes.

Otros participantes relevantes corresponden a los profesores, quienes aparecen con menos protagonismo que los estudiantes y en un rol más ligado al acompañamiento y a los apoyos de las actividades cotidianas. Por una parte, así como los estudiantes se visualizan como gestores de actividades, los docentes aparecen como los acompañantes, incluso en ocasiones como ayudantes, de las actividades referidas en los grupos focales, de tal manera que se produce un intercambio de roles. Por otra parte, el espacio para los profesores emerge en las dimensiones que escapan a las competencias de los estudiantes. La siguiente cita es ilustrativa al respecto:

Ejemplo 5:

Estudiante1: En las actividades del lunes podrían participar todos los alumnos y también los profesores. Los profesores nos [i] podrían ayudar (grupo focal, escuela 2).

Ejemplo 6:

Estudiante1: Si salimos de la escuela o a la playa los profesores [j] pueden participar. Con los chicos es [k] complicado pues se [l] arrancan. Niños de 6to para arriba.

E [grupo 1]: Los niños son [m] complicados.

E [grupo 2]: Pero eso sería [n] injusto, los profes [ñ] pueden ayudar (grupo focal, escuela 2).

En términos valorativos, en el ejemplo 5 apreciamos una valoración positiva de capacidad. Esta valoración [i] focaliza la habilidad de los docentes respecto a los estudiantes sobre la resolución de problemas prácticos en el desarrollo de las actividades que ellos mismos diseñan. Aunado a lo anterior, en el ejemplo 6 emergen los participantes estudiantes de primaria que se caracterizan con valoraciones de integridad negativa acerca de su comportamiento [k] [m] "complicado" y [l] "arrancan"; esto les lleva a pensar la posibilidad de que los estudiantes de primaria no asistan a las salidas pedagógicas, lo que activa la categoría de apreciación negativa de integridad [n].

Esta integridad negativa representada con la "injusticia" es resuelta por los estudiantes mediante la participación directa de los docentes, a quienes se les condiciona su participación en términos funcionales; es decir, se les posiciona con una capacidad de hacer cosas referidas al control u operacionalización de actividades [i] [j] [ñ], en las cuales los docentes resuelven problemas que surjan en las actividades organizadas por el propio estudiantado.

Finalmente, es posible visualizar en los relatos la presencia de otros actores que comúnmente no están en el espacio escolar, como instituciones aledañas, algunos 
considerados "expertos" por los estudiantes o redes de apoyo, junto con breves apariciones de los apoderados, pese a que no son tan frecuentes en relación con el análisis de contenido. Estos actores sociales se mencionan de manera escasa, pero cuando aparecen son valorados. Los estudiantes señalan que les gustaría aprender una multiplicidad de tareas y disciplinas (educación sexual, freestyle o natación, por ejemplo), muchas de las cuales, al parecer, no pueden ser enseñadas por los actores escolares formales. La siguiente cita es representativa y ejemplifica esta participación de actores expertos:

Ejemplo 7:

Monitora: ¿Y quién las enseñaría? [actividad recreativa].

Estudiante1: Alguien que [o] supiera de todo eso, alguien como que fuera un [p] experto (grupo focal, escuela 3).

Sobre el posicionamiento intersubjetivo de los estudiantes, como mencionamos en el análisis de contenido, emerge en el discurso el participante experto, quien se posiciona a través de una capacidad cognitiva o procedimental: el que sabe, el que puede hacer. En el ejemplo 7, el experto irrumpe de forma intensificada: primero es un participante que sabe [o] y luego se configura como experto propiamente tal [p].

De manera menos prominente, identificamos en el discurso a los asistentes de la educación y los apoderados, en el primer caso posicionados desde una perspectiva afectiva y en el segundo, desde una de integridad:

Ejemplo 8:

Estudiante1: ...hacer a los trabajadores, darle un [q] día libre (grupo focal, escuela 4).

Ejemplo 9:

Estudiante1: Los papás deben $[\mathrm{r}]$ medir sus palabras cuando están frente a un niño o cuando [s] nos retan y nos dicen que [t] no hablen disparates y empiezan a hablar ellos (grupo focal, escuela 2).

En los ejemplos 8 y 9 observamos las valoraciones sobre los asistentes de la educación y los apoderados, respectivamente. En el caso de los primeros, se encuentran valoraciones relacionadas con el afecto negativo de satisfacción, es decir, se presentan a los asistentes de la educación como cansados [q]. Este posicionamiento se expresa en otros adultos de la escuela, como los profesores, quienes también son valorados por su cansancio o estrés:

Ejemplo 10:

Estudiante 1:... así los profes descansan, así no tienen tantos problemas (grupo focal, escuela 4).

Además, se expresa una valoración de integridad positiva implícita por parte de los estudiantes al buscar soluciones a esas dificultades que identifican en los adultos de la escuela. Respecto a los apoderados, su aparición como participantes en el discurso surge en todos los casos motivada por preguntas de los monitores. La presencia de los apoderados es siempre negativa o inexistente; en el ejemplo 6 apreciamos 
valoraciones de juicio de integridad negativa en las que se sanciona a los estudiantes. Simultáneamente, se acoplan valoraciones negativas de integridad hacia apoderados con valoraciones negativas de capacidad hacia los estudiantes, ya que la valoración [t] implica una incapacidad de los estudiantes en cuanto a la pertinencia de sus intervenciones en sus respectivos hogares.

En síntesis, la presencia de estudiantes, profesores y personal externo varía en cuanto a su protagonismo en los procesos de participación escolar del alumnado en la escuela, así como en sus roles y funciones. Existen otros actores que se configuran como parciales para los estudiantes, como apoderados y asistentes de la educación. Otros actores, por ejemplo, directivos o líderes medios, no aparecieron en las experiencias escolares de los estudiantes.

\section{¿Cómo desean participar?}

En cuanto a las formas en que los estudiantes desean participar, los procedimientos, estilos y modos de estar en la escuela, es posible identificar tres categorías que configuran los procedimientos antes señalados: el juego como forma de participación; haciendo y aprendiendo; y reinventando las prácticas educativas tradicionales.

El juego, más que una metodología de trabajo, se dispone desde los relatos de los estudiantes como un modo de estar y participar en la escuela. Mientras las actividades sean más lúdicas, entretenidas y estén alejadas de la racionalidad escolar cotidiana, más dialogantes van a ser con la forma deseable que tienen los estudiantes de estar en el espacio escolar. Incluso, algunos estudiantes ven en el juego una manera de compensar las experiencias escolares menos entretenidas, mientras que para otros el juego remedia la ausencia de actividades:

Ejemplo 1:

Podemos poner play para que los niños que [a] nunca han jugado se [b] diviertan más que con lo que hacen todos los días (grupo focal, escuela 4).

En el ejemplo 1 advertimos valoraciones afectivas de insatisfacción [s] "nunca han jugado", la cual se expresa de manera evocada, ya que se infiere que no haber jugado no es divertido. Posteriormente, el no jugar contrasta con el afecto positivo de satisfacción [b], que, su vez, se intensifica "diviertan más". Por otra parte, algunos estudiantes dicen que hay horas vacías y piensan cómo cubrirlas. Una estudiante responde "con juegos" y luego toma la imagen y la pega en el horario (nota de campo, escuela 2).

Otra forma en que los estudiantes desean participar es mediante actividades prácticas de aprendizaje; es decir, haciendo y aprendiendo. La presencia de los estudiantes en la escuela se vuelve compleja:

Ejemplo 2:

Monitora: ¿Cómo a ti te gustaría aprender sobre astronomía?

Estudiante 1: Que me enseñaran [c] leyendo, [d] viendo videos y [e] siguiendo instrucciones (grupo focal, escuela 3). 
Ejemplo 3:

Monitora: ¿Quién les tendría que enseñar?

Estudiante: La profesora, la tía Cintia, que [f] sepa, que le [g] interese, o que hagan un [h] taller de química, o podría ser un [i] taller. Sí porque la [j] clase, para conseguir una sala podría ser [k] difícil, sería [l] complicado (grupo focal, escuela 3).

En términos valorativos, el ejemplo 2 muestra una serie de valoraciones que configuran la idea de lo lúdico o novedoso respecto al aprendizaje. A través de las valoraciones [c], [d] y [e], se expresan apreciaciones de evaluación positiva, que valoran actividades comunes que el estudiante estima lúdicas. El mismo proceso se aplica por medio de quién ejerce la actividad lúdica, como se indica en el ejemplo 3. En este caso, la profesora cumple el rol de experta y se destaca su capacidad [h] e integridad [g].

Sumado a lo anterior, opera una contraexpectativa sobre las voces presentes [i] y [k] acerca de la valoración, que diferencian entre taller y clase. El taller [i] funciona positivamente en términos de apreciación, y la clase [j] de forma negativa a través de la impregnación en cuanto a las valoraciones [k] y [l] de apreciación negativa.

En sintonía con las actividades lúdicas y el aprender haciendo, los estudiantes señalan que lo relevante sería reinventar las prácticas educativas tradicionales de la escuela, en cuanto a transformar la experiencia escolar, cambiar las acciones que nunca fueron consensuadas con los estudiantes o las que se distancian de los principales intereses del alumnado. Dichas actividades pueden ser dentro o fuera de la sala de clases y cambiando los horarios escolares:

Ejemplo 4:

Estudiante: Puede ser que los alumnos [m] ayuden a elegir un libro que a todos les guste, para que a todos les [n] guste y todos [ñ] no estén [o] aburriéndose porque [p] no les gusta (grupo focal, escuela 6).

Ejemplo 5:

Estudiante: Podríamos observar las estrellas en la noche.

Monitor: Alonso, me podría explicar ¿cómo podemos ver las estrellas si estamos de día?

Estudiante: Venimos a la noche a la escuela y hacemos una piyamada en la plaza, eso lo podríamos dejar como un taller.

La valoración del ejemplo 4 resalta la posibilidad de elección y la valoración positiva de afecto sobre la posibilidad de "gustar" [n] y "no aburrirse" [ñ]. Estas valoraciones acerca de los estudiantes operan también sobre la actividad y configuran una apreciación de evaluación positiva cuando la actividad es "entretenida" [m], y negativa cuando es "aburrida" [p]. En tanto, el ejemplo 5 corresponde a otro antecedente sobre la apreciación de los estudiantes en relación con tipos de actividades; aquí la variación de la actividad que se sale del contexto de aula se configura como más lúdica, o más positiva en términos valorativos, y corresponde a un taller.

La forma en que los estudiantes desean participar en la escuela consiste en que el centro escolar ofrezca nuevas experiencias escolares, las cuales no necesariamente las 
tienen que diseñar los adultos, pero sí la formalidad de la cotidianidad debe sufrir cambios y avanzar en juegos, aprendizajes, prácticas e innovación de prácticas educativas.

¿Para qué desean participar los estudiantes?

En este tema obtuvimos tres respuestas: los estudiantes participan para desarrollarse personalmente, mejorar su experiencia escolar y tomar decisiones. En cuanto al desarrollo personal, los estudiantes reconocen una necesidad entre los adultos y entre ellos mismos como adolescentes, la cual podemos precisar como una necesidad de valores. Desde sus voces, desean estar en la escuela y participar en ella para ser buenas personas. Aunque el aprendizaje es una dimensión reconocida, el desarrollo personal es clave en la experiencia escolar. La siguiente cita es representativa:

Ejemplo 1:

Si bien [a] queremos asignaturas de lenguaje o matemáticas, también nos [b] gustaría el desarrollo personal. Que se nos [c] enseñe a respetarnos entre nosotros (grupo focal, escuela 2).

Este desarrollo personal sería funcional en varias dimensiones, entre las cuales destaca el valor de la diversidad. Cuando los estudiantes hablan de "respetarnos entre nosotros", se refieren a la importancia de llevarse bien en la escuela y que todos tengan cabida. Los estudiantes se centran en las personas embarazadas que no pueden hacer todas las actividades de la escuela. Sin embargo, buscan alternativas: "puede cocinar", "puede hacer música", "puede pintar" (nota de campo, escuela 2).

Siguiendo el ejemplo 1, por un lado, los estudiantes se posicionan valorativamente desde afectos positivos de inclinación [a] "queremos", [b] "gustaría”, lo cual se relaciona con el deseo de aprendizaje de ellos mismos. Por otro, esta inclinación hacia un tipo particular de aprendizaje se orienta a una valoración de juicio de integridad negativo [3] "enseñe a respetarnos", ya que se autorrepresentan como carentes o poco respetuosos e indican su intención de desarrollar tal valor.

En lo concerniente al mejoramiento de la experiencia escolar, entendemos que los estudiantes desean cambiar las rutinas típicas de escolaridad provenientes de actividades de aula, horarios estructurados, lógicas de poder adultocéntricas e, incluso, ampliando las posibilidades de aprendizaje. La experiencia escolar mejoraría si se mueven más los límites de la norma y de las actividades comúnmente planificadas por los adultos. En el ejemplo 2 presentamos una serie de fragmentos del corpus:

Ejemplo 2:

Estudiante: Podemos visitar a niños que se le [d] hayan muerto sus familiares, podemos ir [e] todos; se dividan las personas y vayan a los lugares que les [f] gustan; los miércoles se dividen según los cursos, primero A, B, segundo A, B, el primero va a un lugar y el segundo va a otro; podríamos hacer un tenedor libre y nosotros cocinar (nota de campo, escuela 2).

No se cambia la experiencia curricular; más bien se amplía. Los estudiantes mantienen las ganas de aprender matemáticas, lenguaje o ciencias. Sin embargo, desean mejorar la vida en la escuela mediante la incorporación de nuevos saberes e intentando colectivizarlos. En términos valorativos, la identificación de a quiénes se 
ayuda o apoya, implica de forma evocada una valoración positiva de integridad [d]; además, estas valoraciones se intensifican al integrar a "todos" [e] los compañeros, lo cual implica una apertura de voces que da cuenta de que hay más participantes implicados en su relato. Finalmente, se realizan valoraciones de afecto positivo relacionadas con el gusto o la satisfacción [f] de los lugares visitados, vinculadas a criterios de selección de las actividades:

Ejemplo 3:

[Los estudiantes hablan sobre aprender de cocina]... comida y bebestibles, comida sería una asignatura de gastronomía en donde nosotros obviamente haríamos nuestra propia comida y la comeríamos [...] Arte, pero opcional, porque arte igual no es solo pintar, el arte varía muchas cosas, cada uno vería su arte (grupo focal, escuela 5).

Por último, es posible reparar en que los estudiantes desean participar porque esperan tomar decisiones sobre su espacio escolar, su vida en el aula y la comunidad en general. Si bien, la mayoría de las decisiones pasan por las propias experiencias escolares, como en esta cita:

Ejemplo 4:

Estudiante: En ciencia vamos a hacer mezclas, acá haremos igual química, artes, vamos a cambiar de color todos los días, los muros de la escuela (grupo focal, escuela 6).

Ejemplo 5:

Estudiante: Nos gustaría ir a Iquique, ir a la playa, realizando [g] acciones comunitarias, [h] ayudar a la comunidad, [i] reciclaje. Todo el día afuera. Los valores son [j] importantes (grupo focal, escuela 2).

En términos valorativos, el ejemplo 5 da cuenta de cómo una acción calificada como "comunitaria" se carga por saturación de manera positiva; así, la acción se valora como una apreciación de integridad [g], la cual, a su vez, se relaciona con el juicio de integridad de los estudiantes, que son quienes "ayudan" [h] y "reciclan" [i]. Finalmente, el estudiante valora en forma explícita, mediante una apreciación de integridad positiva, el desarrollo de valores. En el ejemplo 6 apreciamos algunos patrones que emergen en la categoría de desarrollo personal:

Ejemplo 6:

Estudiante 1: Yo no estoy diciendo informar sobre eso [comunidad LGTBI], digo [k] instruirlas. Porque a veces hay personas que [l] no se sienten [m]bien porque las [n] molestan aquí y las [ñ] molestan allá (grupo focal, escuela 1).

En el ejemplo 6 advertimos el posicionamiento de uno de los estudiantes respecto al desarrollo del conocimiento sobre la comunidad LGTBI en su escuela, el cual se expresa en el juicio de capacidad positiva intensificado por impregnación [k], en el sentido de que no solo se debe informar, sino instruir a los compañeros, lo que aumenta el significado valorativo sobre el conocimiento del tema. Además, este conocimiento se relaciona con experiencias afectivas relatadas por el estudiante, todas negativas [1], [m] y [n] y concernientes a la insatisfacción. El patrón de un objetivo, en este caso el conocimiento, más que un problema o malestar, es justamente uno de los modos de producción discursiva del desarrollo personal de los estudiantes en la escuela. 
Segundo nivel: dimensión explicativa de la participación estudiantil

A continuación presentamos la dimensión explicativa del análisis a partir del vínculo entre el análisis de contenido y la mirada discursiva sobre la experiencia de los estudiantes.

¿Con quiénes desean participar? Primero los estudiantes, luego los adultos

Los estudiantes se consideran altamente capaces para organizar y producir actividades; esto, a su vez, refiere valores que los estudiantes resaltan sobre sí mismos. En el corpus analizado observamos dos participantes clave: estudiantes y adultos. Los primeros se posicionan como participantes protagónicos a través de roles dinámicos mediante los cuales se creen capaces de organizar y producir actividades en el contexto escolar, así como rescatar valores relevantes en el ámbito social, como la generosidad y la justicia, que los habilitan para ejecutar las acciones que proponen.

Los adultos, por su parte, son presentados por los estudiantes como participantes secundarios. Los profesores y auxiliares son representados como sujetos cansados e insatisfechos, lo cual afecta de manera directa la relación con los estudiantes. Los profesores son requeridos para ejecutar actividades de carácter funcional, como manejar un autobús o controlar la conducta de los estudiantes más pequeños. Sumado a ello, la familia aparece escasamente y valorada con baja capacidad e integridad, es decir, sin conocimiento y con valores diferentes de los que poseen los estudiantes.

La configuración de los participantes dentro del corpus analizado se relaciona con estudiantes autocaracterizados en forma positiva y adultos concebidos de modo negativo. Esto describe un círculo de acciones en el que se identifica a docentes agotados que no pueden llevar a cabo actividades -en palabras de los estudiantes- más "divertidas" o "diferentes". Los docentes no son necesariamente personificados de manera negativa por como son; más bien, por las consecuencias de las condiciones en las cuales se desempeñan. Esta identificación de los participantes en la escuela se enmarca en las lógicas neoliberales y de rendición de cuenta, en las que los profesores actúan como un puente entre los requerimientos de las políticas públicas y de los directivos. En esta misma línea, los estudiantes se presentan eficientes y capaces en su poder de organización y su integridad para efectuar acciones.

¿Cómo desean participar? Sea cual sea la forma, que sea lúdica y novedosa

En el corpus analizado emergen una serie de actividades que los estudiantes identifican como espacios de participación. Estos corresponden a enseñar ciencia en laboratorios, leer y tener clases de música, los cuales se valorizan al rediseñarse como actividades prácticas. Este rediseño implica una mayor apropiación de la actividad y una mayor percepción de participación por parte de los estudiantes. La participación se expresa en que los estudiantes pueden deliberar acerca de las acciones que realizan sobre sí mismos y sobre la comunidad a la cual pertenecen.

Otros modos de llevar a cabo acciones se orientan a romper rutinas escolares, lo que se expresa en propuestas de visitas a la escuela en horarios inesperados, por 
ejemplo, talleres de astronomía en las noches o actividades fuera de la escuela de manera constante y sistemática, como la organización de viajes a la playa. Estas acciones pueden funcionar de dos modos: en suspensión y en reinvención.

Las actividades en suspensión corresponden a acciones escolares que no guardan vínculo directo con el currículo, sino que lo detienen temporalmente, como jugar videojuegos o salir a un paseo. Por su parte, las actividades en reinvención refieren acciones de interpretación del currículo en función de las necesidades de los propios estudiantes.

Estos modos de participación se expresan en forma valorativa a través de posicionamientos desde lo afectivo para determinar lo novedoso o satisfactorio de la actividad en suspensión, o bien, en términos de apreciación positiva de las acciones de actividad de reinvención. En el primer caso, la transformación de la rutina es radical y recae en los estudiantes al mejorar su experiencia escolar; en el segundo caso, la valoración es sobre la actividad escolar más que del estudiante en sí mismo.

El modo en que los estudiantes se expresan sobre la participación muestra un claro interés en la mejora de la experiencia escolar, que, en algunos casos, se enfoca hacia la transformación de una escuela conocida y, en otros, a intentar que la escuela sea otra cosa y funcione con criterios de lo lúdico. En el corpus analizado entendemos la forma de hacer cosas que desean los estudiantes como un posicionamiento afectivo, ya que una actividad típicamente rutinaria puede convertirse en una "actividad mejorada" a partir de la participación y el sentido de pertenencia sobre esta, lo cual se relaciona con la siguiente pregunta.

¿Para qué desean participar? Para desarrollarse personalmente y tomar decisiones

Los estudiantes expresan que las razones de participación son el desarrollo personal, la mejora de experiencia escolar y la posibilidad de tomar decisiones. Sobre el desarrollo personal, se muestran abiertos a cambiar su propio punto de vista sobre la escuela y las actividades que ahí realizan; problematizan situaciones escolares y rescatan aspectos de valor de sus propias experiencias. La experiencia escolar personal se intenta mejorar desde la transformación comunitaria, lo cual se representa con un posicionamiento intersubjetivo, como una autovaloración positiva de la integridad de valor en torno a lo colectivo.

En cuanto a la mejora de la experiencia escolar, los estudiantes buscan cambiar la rutina típica en el contexto de aula y proponen actividades que no son transformadoras; más bien operan como espacios donde las actividades se amplían. Los estudiantes intentan abrir el mundo social en el que se desenvuelven y extender el margen de norma que es impuesto por los adultos.

La posibilidad de dar sentido a las actividades tiene que ver también con la representación de los estudiantes como participantes en sus propios discursos. La participación se carga de capacidad respecto a la operación y producción de actividades escolares, es decir, los estudiantes se representan con la habilidad de realizar las acciones que proponen.

Finalmente, la posibilidad de toma de decisiones se presenta como un fin en sí mismo, como una actividad que es propia de los estudiantes; es decir, que ellos diseñen, 
ejecuten o decidan quién realiza se entiende como un "para qué” transversal a lo largo de la revisión del corpus; por ejemplo, una actividad es valorada como más significativa o más divertida si la decisión es tomada por los estudiantes o por la comunidad en conjunto, lo cual tiene que ver con los modos en que los estudiantes se presentan a sí mismos participando. Al mismo tiempo, el sentido de la actividad que ellos desean llevar a cabo refiere una experiencia individual que se colectiviza rápido con la idea de que, si esto le ocurre a un estudiante, podría afectar a muchos.

\section{DiSCUSIÓN Y CONCLUSIONES}

El objetivo de este estudio fue analizar, desde una perspectiva situada, los procesos de participación escolar en función de la valoración de los estudiantes. Esto implica problematizar las propuestas de avances en inclusión y participación del sistema educativo chileno, que, dado el contexto social, económico y político, ha priorizado la dimensión académica, en especial el logro de aprendizajes curriculares evaluados mediante pruebas estandarizas, las cuales, además, tienen altas consecuencias. Lo anterior ha implicado poner en un rincón, es decir, en un lugar visible, pero periférico y tangencial, uno de los procesos centrales de la inclusión educativa, como lo es la participación de los miembros de la comunidad escolar, y en particular del estudiantado como protagonista del fenómeno educativo.

En este estudio, los procesos de participación escolar fueron explorados con base en tres preguntas orientadoras: ¿con quiénes desean participan los estudiantes? ¿Cómo desean participar? ¿Y para qué desean participan? En cuanto a la primera, los resultados señalan que los estudiantes desean participar en la escuela principalmente con pares estudiantes, y dejan en un nivel secundario a los profesores y en uno periférico a los asistentes de la educación y a los apoderados. Entre ellos se valoran de manera positiva y se ven como actores transformadores, y valoran en forma negativa a los adultos, quienes son configurados como actores agotados.

En cuanto a la segunda pregunta, los estudiantes desean participar de forma lúdica, desrutinizando la vida escolar y reelaborando las actividades cotidianas. Aprecian las asignaturas tradicionales siempre y cuando se acompañen de procedimientos entretenidos. La tercera pregunta reveló que desean participar para desarrollarse personalmente en valores, inclusión y diversidad; para ello es relevante la toma de decisiones a fin de transformar la escuela y ampliar los límites de esta.

La participación y discusión dialógica de toda la comunidad educativa se enmarca en un contexto social mayor. Los distintos miembros de la comunidad educativa toman decisiones a partir de las posibilidades que ofrecen las políticas públicas y el marco cultural en el que funciona, y al hacerlo, refuerzan o se resisten a ellos. Las valoraciones de los estudiantes reflejan que la cultura de la rendición de cuentas en la escuela y sus efectos en los miembros adultos de la comunidad no son inadvertidos; ellos reconocen un necesario descanso para los adultos involucrados en su experiencia escolar, así como una rigidez y exceso de rutina que ven capaces de quebrar.

A pesar de que la propia cultura, tanto la escolar, familiar y su entorno, destaca lo académico y coloca el esfuerzo en el centro de su formación escolar (Cornejo y Reyes, 2008), los estudiantes hacen hincapié en los valores que les interesa desarrollar. Mencionan la solidaridad, la preocupación por otros y la generosidad, que 
hacen evidente la posible influencia de medios de comunicación y fenómenos de globalización cultural a través de los cuales promueven actitudes en cuanto al enfoque de derechos de las personas y la no discriminación.

Al mismo tiempo, sus discursos son construidos cultural y socialmente, y expresan ideas novedosas que no son inmunes a las determinaciones sociales, y no solo emergen de una determinada generación, sino desde sus condiciones de clase, género y etnicidad, como sujetos complejos insertos en relaciones sociales diversas (Vergara et al., 2015).

Los hallazgos sugieren que los estudiantes de este estudio de caso tienen una posición definida frente a sus escuelas, que consideran desgastadas. Desde sus posicionamientos intersubjetivos, muestran a adultos cansados y agobiados, a estudiantes aburridos y abrumados. No obstante, reclaman y exigen una mayor presencia en los espacios escolares, y apuestan por una vida escolar más democrática e inclusiva, que confíe en ellos y en su capacidad de aprender y aportar a una transformación de la escuela.

Las propuestas de los estudiantes para cambiar estas escuelas desgastadas no tienen las características de ser radicales, incluso muchas de sus sugerencias son actividades que ya se pueden encontrar en los espacios escolares. Esto demuestra que los estudiantes no desean salir de sus escuelas; más bien involucrarse activamente para mejorarlas (Graham et al., 2018; Lin, 2013). Los hallazgos muestran que los compromisos de los estudiantes con las posibilidades de mejora de su experiencia escolar no son, como se suele pensar, individuales, sino colectivos y guardan relación con el espacio social donde se encuentran; proponen romper jerarquías para que estudiantes, profesores, auxiliares de aseo y profesionales trabajen y aprendan juntos en sociedad, en vez de unos utilizando a los otros para objetivos encubiertos (Nieto y Portela, 2008).

Las escuelas desgastadas son pensadas por el mundo adulto (Lay-Lisboa et al., 2018; Vergara et al., 2015; Poblete y Galaz, 2017; Ascorra, López y Urbina, 2016). Por ende, los procedimientos para escolarizar la infancia que han sido diseñados y aplicados por personas adultas resultan un obstáculo para los sueños de los estudiantes. En su proyección sobre la escuela soñada, buscan más allá de espacios protegidos y diseñados por los adultos desde un paternalismo moderno (Liebel, 2007). Los adultos de la escuela no solo dan continuidad al oficio de alumno o alumna al moldearlos según sus expectativas, sino también al prepararlos para ser un buen alumno o alumna (Perrenound, 2009) según las expectativas sociales y culturales, y también al oficio del profesor, en un rol estático y antidemocrático (López, 2012).

Estas expectativas, como señalamos, se enmarcan en las condiciones sociales, económicas y políticas actuales, en las cuales ser un "buen alumno" en un sistema de "educación de calidad" se define en función de estandarización, desempeño individual y competencia en el sistema escolar. En este contexto, creemos que la participación estudiantil como proceso inclusivo genuino ha quedado postergada a la luz de la importancia de otros ámbitos considerados esenciales y urgentes en educación por los adultos y la sociedad completa, como los resultados académicos medidos con pruebas estandarizadas con altas consecuencias aplicadas a los distintos actores escolares. 
En otras palabras, los cambios culturales que han producido las medidas trasladadas desde la empresa privada a la escuela no dan espacio a la participación de ningún actor escolar, menos de los estudiantes. Según Lerena y Trejos (2015), la filosofía de base de las políticas de mercado ha originado que las escuelas estén más segregadas, aisladas, presionadas y que predomine el sentido de lo privado sobre lo público, lo que hace más difícil la educación comunitaria, una de las características de la educación inclusiva.

En otras palabras, que los adultos e incluso los mismos jóvenes consideren (o no) la voz de los estudiantes es una decisión ligada a una estructura hegemónica, que en el caso chileno -y por supuesto también para Latinoamérica-está representada de manera extrema por la incorporación de principios neoliberales en las políticas educativas (Sisto, 2019) y en las mutaciones culturales que han impactado la escuela (Reyes et al., 2010). Las escuelas desgastadas se encuentran insertas y son, a la vez, efecto del modelo neoliberal llevado a todos los ámbitos sociales, incluida la educación.

En tal sentido, escuchar a los estudiantes y conocer su experiencia escolar y sus sueños respecto a las posibilidades de las escuelas es relevante en el contexto chileno. Los estudiantes desean asumir un rol de codiseñadores/as de espacios de aprendizaje o transformaciones sociales. Reconocemos que esta participación es una oportunidad para ampliar los espacios de democracia en la escuela, y hacer parte a todos los actores escolares. En la medida que avancemos hacia una participación crítica y genuina, las mismas sobreexigencias de los profesores, por ejemplo, se verían beneficiadas, ya que el poder tendría mayores canales de fluidez al expandir la participación comunitaria. Esto, sin duda, es una dimensión que merece mayor atención y exploración.

Avanzar en la participación de los estudiantes implica repensar tanto los espacios escolares como los de investigación. En cuanto a los primeros, la participación sería la puerta de entrada al protagonismo infantil y juvenil escolar, el cual debe ser crítico para no reproducir las desigualdades e injusticias sociales. Respecto a los espacios de investigación, pareciera ser que las dificultades tienen más que ver con los enfoques de investigación que con los niños mismos (Vergara et al., 2015). El desafío sería adoptar enfoques y prácticas de investigación en contra de aquellas dominantes, neoliberales y basados en el mercado (Fielding, 2011). La participación estudiantil, como proceso inclusivo, permitiría dejar en evidencia que las prácticas neoliberales agrietan las posibilidades de democracia en la escuela.

Finalmente, es necesario tener en cuenta el momento histórico y contingente que se lleva a cabo en los procesos sociales en Chile. El 18 de octubre de 2019 se desató un estallido social que buscó transformar lo que popularmente se expresa como "el modelo" implantado por la fuerza desde la dictadura cívico-militar de Pinochet. Parte de las demandas se vinculan de manera directa con la participación educativa y los contextos estructurales de las escuelas -y también universidades-. En forma sistemática, se ha ido configurando al estudiantado como un actor "menor" en el espacio social; en el marco del 18 de octubre estudiantes secundarios dieron el primer paso en la expresión del malestar social a través del espacio que les quedaba de participación. Hoy, en la crisis sociosanitaria del covid-19, el rol del estudiantado vuelve a cambiar y es necesario repensarlo y discutirlo. 
Como proyecciones, es posible señalar que los desafíos se levantan en dos planos: en el escolar y en el investigativo. En cuanto al primero, las actuales condiciones educativas no han dejado espacio a la escucha y toma de decisiones basadas en las perspectivas de los actores escolares. Las comunidades escolares deben discutir los dilemas que exigen las políticas públicas y, en estos debates, ofrecer experiencias escolares con total consideración de las voces de niños, niñas y jóvenes como actores sociales, sea en espacios formales e informales.

En cuanto al ámbito investigativo, es necesario que quienes realizamos estudios en el ámbito educativo consideremos la voz estudiantil como legítima de la experiencia escolar, aspecto que pudimos visualizar en el trabajo de campo, en el cual los participantes se organizaron y apropiaron de la actividad al acordar propuestas sobre la escuela y sus otras posibilidades. Uno de nuestros desafíos próximos es llevar estos resultados a los participantes del estudio y discutir junto con ellos cada una de las preguntas orientadoras y los hallazgos clave de este estudio.

\section{REFERENCIAS BIBLIOGRÁFICAS}

Albornoz, N., Silva, N. y López, M. (2015). Escuchando a los niños: significados sobre aprendizaje y participación como ejes centrales de los procesos de inclusión educativa en un estudio en escuelas públicas en Chile. Estudios Pedagógicos, vol. 41, pp. 81-96. https://dx.doi.org/10.4067/S071807052015000300006

Ascorra, P., López, V. y Urbina, C. (2016). Participación estudiantil en escuelas chilenas con buena y mala convivencia escolar. Revista de Psicología, vol. 25, núm. 2, pp. 1-18.

Assaél, J., Acuña, F., Contreras, P. y Corbalá, F. (2014). Transformaciones en la cultura escolar en el marco de la implementación de políticas de accountability en Chile. Un estudio etnográfico en dos escuelas clasificadas en recuperación. Estudios Pedagógicos, vol. 40, núm. 2, pp. 7-26.

Bajtín, M. (2015). Yo también soy (fragmentos sobre el otro). Buenos Aires: Ediciones Godot.

Barrios, A., Gutiérrez, N., Mayorga, C. y Urrutia, E. (2014). Métodos óptimos para determinar validez de contenido. Educación Médica Superior, vol. 28, núm. 3, pp. 547-558. https://bit.ly/36ZM2X5

Bellei, C. (2015). El gran experimento. Mercado y privatización de la educación chilena. Santiago de Chile: LOM Ediciones.

Booth, T. y Ainscow, M. (2015). Guía para la educación inclusiva. Desarrollando el aprendizaje y la participación en los centros escolares. Adaptación de la $3^{a}$ edición revisada del Index for Inclusion. España: OEI.

Canales, M. (2006). Metodologías de investigación social. Santiago de Chile: LOM Ediciones.

Chávez, P. y Vergara, A. (2017). Ser niño y niña en el Chile de hoy. La perspectiva de sus protagonistas acerca de la infancia, la adultez y las relaciones entre padres e hijos. Santiago: Ediciones Ceibo.

Cornejo, R. y Reyes, L. (2008). La cuestión docente: Chile, experiencias organizacionales y acción colectiva de profesores. Buenos Aires: Laboratorio de Políticas Públicas. Foro Latinoamericano de Políticas Públicas. 
Decreto 291. Diario Oficial de la República de Chile, Santiago, Chile, 13 de julio de 1999.

Denzin, N. \& Lincoln, Y. (2013). The strategies of qualitative inquiry. Londres: SAGE Publications.

Fairclough, N (2013). Critical discourse analysis. The critical study of language. Nueva York: Routledge.

Fielding, M (2011). La voz del alumnado y la inclusión educativa: una aproximación democrática radical para el aprendizaje intergeneracional. Revista Interuniversitaria de Formación del Profesorado, vol. 70, núm. 1, pp. 31-61.

Flick, U. (2012). Introducción a la investigación cualitativa. Madrid: Morata.

Gaitán, L. (2006). La nueva sociología de la infancia. Aportaciones de una mirada distinta. Política y Sociedad, vol. 43, núm. 1, pp. 9-26.

Graham, A., Bessell, S., Adamson, E., Truscott, J., Simmons, C., Thomas, N., Gardon, L. \& Johnson, A. (2018). Navigating the ambiguous policy landscape of student participation. Journal of Education Policy, vol. 34, núm. 6, pp. 789811. doi: 10.1080/02680939.2018.1527945

Halliday, M. y Matthiessen, C. (2014). An introduction to functional grammar. Nueva York: Routledge.

Huang, J. (2019). Being and becoming: The implications of different conceptualizations of children and childhood in education. Canadian Journal for New Scholars in Education, vol. 10, núm. 1, pp. 99-105.

Lay-Lisboa, S., Araya-Bolvarán, E., Marabolí-Garay, C., Olivero-Tapia, G. y Santander-Andrade, C. (2018). Protagonismo infantil en la escuela. Las relaciones pedagógicas en la construcción de ciudadanía. Sociedad e Infancias, vol. 2, pp. 147-170. https://doi.org/10.5209/SOCI.59474

Lerena, B. y Trejos, J. (2015). Sobre la posibilidad de una educación inclusiva bajo el actual modelo de desarrollo económico y social chileno. Revista Latinoamericana de Educación Inclusiva, vol. 9, núm. 2, pp. 145-160.

Ley 19284. Diario Oficial de la República de Chile, Santiago, Chile, 05 de enero de 1994.

Ley 20248. Diario Oficial de la República de Chile, Santiago, Chile, 25 de enero de 2008.

Ley 20201. Diario Oficial de la República de Chile, Santiago, Chile, 06 de junio de 2007.

Ley 20422. Diario Oficial de la República de Chile, Santiago, Chile, 03 de febrero de 2010.

Ley 20529. Diario Oficial de la República de Chile, Santiago, Chile, 27 de agosto de 2011.

Ley 20845. Diario Oficial de la República de Chile, Santiago, Chile, 29 de mayo de 2015.

Liebel, M. (2007). Paternalismo, participación y protagonismo infantil. En Y. Corona y M. E. Linares (coords.). Participación infantil y juvenil en América Latina (pp. 113-146). México: Edición Universidad Autónoma Metropolitana.

Lin, A. (2013). Citizenship education in American schools and its role in developing civic engagement: a review of the research. Educational Review, vol. 67, núm. 1, pp. 1-29. doi: 10.1080/00131911.2013.813440 
López Melero, M. (2012). La escuela inclusiva: una oportunidad para humanizarnos. Revista Interuniversitaria de Formación del Profesorado, vol. 26, núm. 2, pp. 131-160.

López, V., González, P., Manghi, D., Ascorra, P., Oyanedel, J. C., Redón, S. y Salgado, M. (2018). Políticas de inclusión educativa en Chile: tres nudos críticos. Archivos Analíticos de Políticas Educativas, vol. 26, núm. 157, pp.1-18. http:// dx.doi.org/10.14507/epaa.26.3088

Martin, J. \& Rose, D. (2007). Working with discourse. Meaning beyond the clause. Londres/Nueva York: Continuum.

Martin, J. \& White, P. (2005). The language of evaluation. Appraisal in English. Nueva York: Palgrave Macmillan.

Ministerio de Educación (2005). Política Nacional de Educación Especial. Nuestro compromiso con la diversidad. Santiago de Chile: Mineduc.

Nieto, J. y Portela, A. (2008). La inclusión de la voz del alumnado en el asesoramiento para la mejora de las prácticas educativas. Revista Profesorado, vol. 12, núm. 1, pp. 1-26.

Oteíza, T. (2019). Sistema de valoración como herramienta teórico-metodológica para el estudio social e ideológico del discurso. Logos: Revista de Lingüística, Filosofía y Literatura, vol. 29, núm. 2, pp. 207-229.

Perrenoud, P. (2006). El oficio de alumno y el sentido del trabajo escolar. Madrid: Editorial Popular.

Poblete, R. y Galaz, C. (2017). Aperturas y cierres para la inclusión educativa de niños/as migrantes en Chile. Estudios Pedagógicos, vol. 43, núm. 3, pp- 239257. https://dx.doi.org/10.4067/S0718-07052017000300014

Ramírez, L. (2020). Significados, valoraciones y des/legitimaciones discursivas de adolescentes acerca del bienestar en la escuela desde una perspectiva de justicia social (tesis doctoral). Pontificia Universidad Católica de Valparaíso, Chile.

Ramírez, L. y Valdés, R. (2019). El "CASO" como dispositivo de las prácticas escolares. Revista Electrónica Educare, vol. 23, núm. 3. Recuperado de https:// www.redalyc.org/articulo.oa?id=1941/194161290012

Reyes, L., Cornejo, R., Arévalo, A. y Sánchez, R. (2010). Ser docente y subjetividad histórica en el Chile actual: discursos, prácticas y resistencias. Polis, núm. 9, vol. 27, pp. 269-292. doi: 10.4067/S0718- 65682010000300012

Sisto, V. (2019). Inclusión "a la chilena”. La inclusión escolar en un contexto de políticas neoliberales avanzadas. Archivos Analíticos de Políticas Educativas, vol. 27, núm. 23. http://dx.doi.org/10.14507/epaa.27.3044

Slee, R. (2013). How do we make inclusive education happen when exclusion is a political predisposition? International Journal of Inclusive Education, vol. 17, núm. 8, pp. 895-907.

Thousand, J., Villa, R. y Nevin, A. (2015). El rol de los estudiantes como co-enseñantes. Revista Latinoamericana de Educación Inclusiva, vol. 9, núm. 2, pp. 111-126.

Unesco (2018). Guía para asegurar la inclusión y la equidad en la educación. Francia.

Unesco (1994). Declaración de Salamanca y marco para la acción. Salamanca, España. 
Valdés, R. (2019). Prácticas de liderazgo de equipos directivos en el desarrollo de culturas inclusivas en escuelas de la Región de Valparaíso (tesis doctoral). Pontificia Universidad Católica de Valparaíso, Chile.

Vergara, A., Peña, M., Chávez, P. y Vergara, E. (2015). Los niños como sujetos sociales: el aporte de los nuevos estudios sociales de la infancia y el análisis crítico del discurso. Psicoperspectivas, vol. 14, núm. 1, pp. 55-65. doi: 10.5027/ psicoperspectivas-vol14-issue1- fulltext-544 